\title{
Quantifying greenhouse gas emissions
}

\section{Matthias Jonas, et al. [full author details at the end of the article]}

Received: 22 March 2019 / Accepted: 8 April 2019 / Published online: 23 May 2019

(C) The Author(s) 2019

\begin{abstract}
The assessment of greenhouse gases (GHGs) and air pollutants emitted to and removed from the atmosphere ranks high on international political and scientific agendas. Growing international concern and cooperation regarding the climate change problem have increased the need to consider the uncertainty in inventories of GHG emissions. The approaches to address uncertainty discussed in this special issue reflect attempts to improve national inventories, not only for their own sake but also from a wider, system analytic perspective. They seek to strengthen the usefulness of national emission inventories under a compliance and/or global monitoring and reporting framework. The papers in this special issue demonstrate the benefits of including inventory uncertainty in policy analyses. The issues raised by the authors and featured in their papers, along with the role that uncertainty analysis plays in many of their arguments, highlight the challenges and the importance of dealing with uncertainty. While the Intergovernmental Panel on Climate Change (IPCC) clearly stresses the value of conducting uncertainty analyses and offers guidance on executing them, the arguments made here in favor of performing these studies go well beyond any suggestions made by the IPCC to date. Improving and conducting uncertainty analyses are needed to develop a clear understanding and informed policy. Uncertainty matters and is key to many issues related to inventorying and reducing emissions. Considering uncertainty helps to avoid situations that can create a false sense of certainty or lead to invalid views of subsystems. Dealing proactively with uncertainty allows for the generation of useful knowledge that the international community should have to hand while strengthening the 2015 Paris Agreement, which had been agreed at the 21st Conference of the Parties to the United Nations Framework Convention on Climate Change (UNFCCC). However, considering uncertainty does not come free. Proper treatment of uncertainty is demanding because it forces us to take the step from "simple to complex" and to grasp a holistic system view. Only, thereafter, can we consider potential simplifications. That is, comprehensive treatment of uncertainty does not necessarily offer quick or easy solutions for policymakers. This special issue brings together 13 papers that resulted from the 2015 (4th) International Workshop on Uncertainty in Atmospheric Emissions, in Cracow, Poland. While they deal with many different aspects of the uncertainty in emission estimates, they are guided by the same principal question: "What GHGs shall be verified at what spatio-temporal scale to support conducive legislation at local and national scales, while ensuring effective governance at the global scale?" This question is at the heart of mitigation and adaptation. It requires an understanding of the entire system of GHG sources and sinks, their spatial characteristics and the temporal scales at which they react and interact, the uncertainty (accuracy and/or precision)
\end{abstract}


with which fluxes can be measured, and last but not least, the consequences that follow from all of the aforementioned aspects, for policy actors to frame compliance and/or global monitoring and reporting agreements. This bigger system context serves as a reference for the papers in the special issue, irrespective of their spatio-temporal focus, and is used as a guide for the reader.

Keywords Greenhouse gas emissions · Emission inventory · Uncertainty · Paris Agreement · Verification $\cdot$ Monitoring toward reporting $\cdot$ Monitoring toward compliance

\section{Introduction}

This special issue has been compiled to enhance understanding of the uncertainty in estimating greenhouse gas (GHG) emissions and to provide guidance on dealing with the challenges resulting from those uncertainties. Such challenges include, but are not limited to the following: (i) accurately and precisely accounting for emissions in space and time (and their verification); (ii) complying with emission reduction commitments under uncertainty; (iii) dealing with the risk of exceeding future temperature targets; (iv) evaluating mitigation - both domestic and in other countries - versus adaptation in the presence of uncertainty; and (v) trading inherently uncertain emission permits.

The special issue brings together 13 contributions that originated as short papers presented at the 2015 (4th) International Workshop on Uncertainty in Atmospheric Emissions, in Cracow, Poland. Their common concern is to highlight issues where our understanding of uncertainty still falls short. With the momentum of that Workshop, these short papers have been developed further and expanded considerably. All have undergone two to three rounds of improvement, and the short papers have matured into the longer, peer-reviewed papers that are presented here.

\subsection{The rationale for estimating GHG emissions and removals}

The assessment of GHGs and air pollutants (indirect GHGs) emitted to and removed from the atmosphere ranks high on scientific and political agendas, which is steered internationally by the Intergovernmental Panel on Climate Change (IPCC) on the one hand, and the United Nations Framework Convention on Climate Change (UNFCCC) on the other.

The UNFCCC provides the foundation for multilateral action to combat climate change and its impacts on humanity and ecosystems. Its objective is to "stabilize greenhouse gas concentrations in the atmosphere at a level that would prevent dangerous anthropogenic interference with the climate system." In pursuit of this objective, the UNFCCC established a framework with broad principles, general obligations, basic institutional arrangements, and an intergovernmental process for agreeing to specific actions over time, supported by international instruments such as the 1997 Kyoto Protocol (in force until 2020) and its successor, the 2015 Paris Agreement (in force as of 2016).

The Paris Agreement aims to keep this century's mean global temperature rise well below $2{ }^{\circ} \mathrm{C}$ above pre-industrial levels and to pursue efforts to limit the temperature increase to $1.5^{\circ} \mathrm{C}$. The Agreement requires all signatory countries to employ their best efforts to achieve this goal through nationally determined contributions and to strengthen their efforts in the 
years ahead (see also Tollefson 2018a, b). The Agreement includes the requirement that all signatory countries report regularly on their emissions and implementation efforts. A global stock-take will be carried out every five years to assess collective progress toward achieving the aims of the Agreement and to inform further actions by individual countries (UNFCCC 2018).

GHG emissions are rarely measured directly. To assist countries in compiling comprehensive emission inventories and conducting quantitative uncertainty analyses under the UNFCCC, the IPCC has put forward standardized methodologies to enable adequate accounting of national, natural, and human-induced GHG sources and sinks (IPCC 2000, 2006). Policymakers use these national inventories to develop strategies and policies for emission reductions and to track the progress of these policies (cf. also Box 1). Where formal commitments exist, regulatory agencies and corporations rely on inventories to establish compliance records. Businesses, the public, and other interest groups use inventories to better understand the sources and trends in emissions.

Box 1 Rationale for using the UNFCCC to report national GHG emissions. Source: NRC (2010: Summary); modified

The UNFCCC framework for reporting national emissions comprises three main elements:

- an internationally negotiated and accepted capability to monitor the national anthropogenic emissions of the most important GHGs;

- an independent review by an international body to determine whether appropriate procedures and methods are being used to prepare national inventories, identify inconsistencies within and between inventory reports, and to take action if problems are uncovered; and

- an established mechanism through the IPCC to incorporate new information and to strengthen inventory methods.

\subsection{The rationale for conducting uncertainty analyses}

GHG inventories contain uncertainty for a variety of reasons. The current policy approach of ignoring inventory uncertainty altogether (inventory uncertainty was/is monitored, but not regulated, under the Kyoto Protocol) is problematic. Emission reductions are activity- and gas-dependent and can range widely, which means that sufficient and appropriate data are required. Biases (discrepancies between true and reported emissions) are not uniform across space and time and can discredit flux-difference accounting schemes, which tacitly assume that biases are canceled out. The human impact on nature is not necessarily constant and/or negligible, and this can jeopardize a partial-system (partial, hereafter) GHG accounting approach that is not a logical subset of a full-system (full, hereafter) GHG accounting approach, and not safeguarded by one. Full accounting allows for the shortcoming of inventories, rooted in the bottom-up accounting of emissions, to be overcome. Even for relatively well-constrained industrial GHGs, global emissions based on top-down methods (using atmospheric measurements) often agree poorly with the bottom-up emissions reported (e.g., Weiss and Prinn 2011). Being aware of the uncertainties involved, including those resulting from our system view, will help to strengthen future political decision-making.

The rationale for conducting and improving uncertainty analyses elaborated by White et al. (2011: 3-18) in the wake of the 2010 (2nd) Uncertainty Workshop appears to still be valid (cf. also Box 2). 
Box 2 Rationale for improving and conducting uncertainty analyses. Source: White et al. (2011: 3-18); modified

- Calculations of GHG emissions contain uncertainty for a variety of reasons such as lack of availability of sufficient and appropriate data and of techniques for processing them.

- To understand the basic science of GHG gas sources and sinks, an understanding of the uncertainty in their estimates is required.

- Schemes to reduce human-induced global climate impacts rely on the confidence that inventories of GHG emissions allow emissions and emission changes to be accurately assessed. To ensure such confidence, it is vital for the uncertainty present in emissions estimates to be transparent. Clear communication of the forces underlying inventory uncertainty may be needed so that their implications are understood.

- Uncertainty estimates are not necessarily intended to dispute the validity of national GHG inventories, but they can help improve them.

- Uncertainty is higher for some aspects of a GHG inventory than for others. For example, past experience shows that, in general, methods used to estimate nitrous oxide $\left(\mathrm{N}_{2} \mathrm{O}\right)$ emissions are more uncertain than methods to estimate methane $\left(\mathrm{CH}_{4}\right)$, and much more uncertain than those for estimating carbon dioxide $\left(\mathrm{CO}_{2}\right)$ emissions. If uncertainty analysis is to play a role in cross-sectoral or international comparison or in trading systems or compliance mechanisms, then approaches to uncertainty analysis need to be robust and standardized across sectors and gases, as well as among countries.

- Uncertainty analysis helps to understand uncertainties: science helps to reduce them. Better science needs support, encouragement, and greater investment. Full carbon accounting (FCA), in other words, full accounting of emissions and removals in national GHG inventories, including all GHGs, is important for advancing the science.

- FCA is a prerequisite for reducing uncertainties in our understanding of the global climate system.

From a policy viewpoint, FCA could be encouraged by including it in reporting commitments, but it could be separated from negotiations on reduction targets. Future climate agreements will be made more robust by explicitly accounting for the uncertainties associated with emission estimates.

\section{What did we know about uncertainty?}

The papers in this special issue address uncertainty from a wide range of perspectives, as outlined in detail below. This leads to an important question as to the advances presented by the authors. Below, we provide a summary of the status quo of uncertainty research as it was perceived prior to the 2015 Uncertainty Workshop. Before that, six interdependent key insights had materialized which, according to experts, were considered to need further research. The insights centered around (cf. Ometto et al. 2015: v-xii; modified):

1. Verification: reconciling bottom-up and top-down accounting of GHG emissions.

The bottom-up approach to accounting currently used for GHG emissions is incomplete in itself, as it cannot deal with the issue of accuracy. Bottom-up accounting of emissions is important in the sense that it shows which activities and actors are responsible for emissions. However, the ultimate accounting must be directed top-down, and reductions in emissions must be reflected in reductions in atmospheric GHG concentrations.

2. Avoiding systemic surprises: distinguishing between subsystems with fundamentally different characteristics related to emission dynamics and uncertainty before they are superimposed.

Earth's ecology acts as a complex and nonlinear system that is in a constant state of change. This system can be best understood when taking a long-term perspective. One should not expect to utilize nature to reduce anthropogenic GHG emissions in the same way that we use technological opportunities. By anticipating some accounting pitfalls, we can state that, to avoid surprises, we need to exercise caution in superimposing subsystems with different characteristics related to emission dynamics and uncertainty. 
3. Making uncertainty analysis a key component of national GHG inventory analysis to support the development of informed policy in the framing of international environmental agreements: providing advanced guidance beyond the methodologies offered by the IPCC to ensure uncertainty is dealt with appropriately in an internationally consistent way across countries, subsystems, sources and sinks, GHGs, and sectors.

Uncertainty analysis should be used to develop clear understanding and informed policy to support the framing of international environmental agreements. To ensure that uncertainty analysis becomes a key component of national GHG inventory analysis in support of international environmental policy, advanced guidance is needed so that uncertainty can be dealt with appropriately in an internationally consistent way across countries, subsystems, sources and sinks, GHGs, and sectors. This guidance goes beyond the methodologies offered by the IPCC to conduct and execute uncertainty analyses.

4. Minimizing the impact of uncertainty to support the design of advanced policy agreements: providing approaches that allow subsystems to be treated individually and differently rather than collectively (in terms of $\mathrm{CO}_{2}$-equivalence) and equally (not distinguishing between emissions and removals).

Uncertainty is higher for some GHGs and some sectors of an inventory than for others. Nature-related emissions and removals (e.g., in the land use, land-use change, and forestry (LULUCF) sector and in the landfill sector) have greater uncertainty than technospheric emissions (e.g., in the fossil-fuel sector); and current estimates of Nitrogen (eg, $\mathrm{N}_{2} \mathrm{O}$ ) emissions are more uncertain than methane $\left(\mathrm{CH}_{4}\right)$ and $\mathrm{CO}_{2}$ emissions. This raises the option that, when future policy agreements are being designed, some components of a GHG inventory could be treated differently from others. The approach of treating subsystems individually and differently would allow emissions and uncertainty to be looked at simultaneously and would thus allow for differentiated emission reduction policies. This approach could have an advantage over treating all GHG emissions and removals collectively (in terms of $\mathrm{CO}_{2}$-equivalence) and equally (not distinguishing between emissions and removals), which usually leads to increased uncertainty, with potentially important scientific and policy implications (e.g., in cases where countries claim to have fulfilled their commitments to reduce or limit emissions). To recall, under the Kyoto Protocol, the agreed emission changes for most countries were of the same order of magnitude as the uncertainty that underlay their combined emission estimates.

5. Full GHG accounting: ensuring that any differentiated approach to accounting forms a logical subset of a full GHG accounting approach.

Any differentiated approach to accounting must form a logical subset of a full GHG accounting approach. Full accounting is the only way to reach a proper understanding of the global climate system and is a prerequisite for reducing the uncertainties in that understanding. Providing reliable and comprehensive estimates of uncertainty cannot necessarily be achieved by applying the approach favored under the UNFCCC and the Kyoto Protocol, which provided only for partial accounting of GHG fluxes to and from the atmosphere. It is virtually impossible to estimate the reliability of any system output if only part of the system is considered.

6. Compliance versus reporting (bifurcation of agreements) but in a complementary manner: providing options that allow for smarter treatment of subsystems, for example, individually and differently, while at the same time following full GHG accounting.

The option of treating subsystems individually and differently, while at the same time following full GHG accounting, forces us to deal with subsystems more skillfully than we 
have in the past. The maxim to follow would be to treat the technosphere, our built environment, and the biosphere individually, but also holistically. Dealing with the technosphere and biosphere individually and differently, but not independently, although leading to agreement bifurcation, has clear advantages for emission inventories. First, it does not jeopardize verification - atmospheric measurements can discriminate between fossil fuel, terrestrial biosphere, and ocean carbon by means of their carbon isotope fingerprints in combination with measurement of atmospheric oxygen $\left(\mathrm{O}_{2}\right)$; but they cannot identify individual fluxes within any of these categories. Second, differentiated accounting offers the option of (i) placing emissions from the technosphere, where uncertainty is believed to be lowest, under stringent compliance with clear rules for dealing with uncertainty, while (ii) putting biospheric emissions and removals, with their greater uncertainties, under consistent reporting by means of a global monitoring framework.

\section{What do we know about uncertainty today and what do we report on in this special issue?}

The papers in this special issue all agree that dealing with uncertainty is not a quick exercise but rather involves a commitment that is painstaking and long-term. Proper treatment of uncertainty is costly in terms of both time and effort because it forces us to take the step from "simple" to "complex" in order to grasp a wider and more holistic system view. Only after we have taken that step, can we consider simplifications that may be warranted.

To facilitate visualization of the 13 papers within a system context, we group them using a matrix (cf. Table 1). Vertically, the matrix relates to the IPCC's classification of sectors of

Table 1 Visualization of the scientific context of the 13 papers in this special issue. All papers refer to $\mathrm{CO}_{2}$ unless indicated otherwise by " $\mathrm{CO}_{2}$-eq"

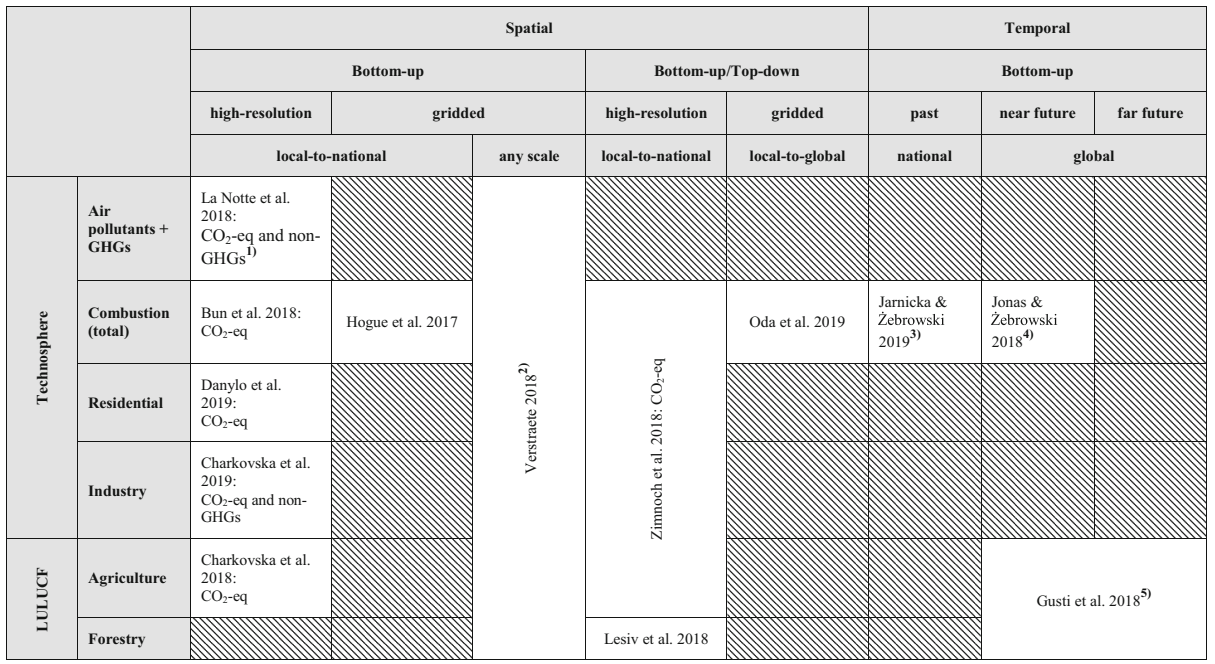

1) Inventory support: beyond GHG reporting. ${ }^{2)}$ Methodological support: regridding. ${ }^{3)}$ Methodological support: learning. ${ }^{4)}$ Methodological support: explainable outreach. ${ }^{5)}$ Methodological support: sensitivity of costs. Matrix cells without an entry are to be understood as "not addressed here." 
emissions and removals (see the first two columns from the left); while horizontally, it lists the systemic features that are most pertinent to the papers (see the first three rows from the top). All papers refer to $\mathrm{CO}_{2}$ unless indicated otherwise (by " $\mathrm{CO}_{2}$-eq"). Two groups of papers are distinguished according to whether they follow a bottom-up accounting approach in addressing uncertainty (Group I) or a bottom-up/top-down one (Group II). Five of the 13 papers are content-specific and are clustered under Group III; they provide beyond-inventory or methodological support to improve our understanding and handling of uncertainty (see the footnotes below Table 1).

We use Table 1 to guide readers through the papers and the scientific advances reported on in this special issue and to describe how they contribute to the key insights/issues (1-6) mentioned in the previous section - and of the likely consequences should these insights be heeded. Together, all papers confirm or advance key insights 1, 3, 4, and 5 .

Matrix cells without an entry are to be understood as "not addressed here". Interested readers are directed to the outcomes of earlier Uncertainty Workshops (http://www.ibspan. waw.pl/unws2015/index.php?go=home) or to the scientific literature.

\subsection{Group I: Bottom-up accounting (high resolution or gridded)}

The papers by Bun et al. (2018), Charkovska et al. (2018, 2019), Danylo et al. (2019), and Hogue et al. (2017) address bottom-up accounting, high-resolution or gridded. They contribute to key issue 1 (one-sided perspective), contribute substantially to key issue 3 , and advance key issue 4 .

Two papers are close in content to this group, although listed elsewhere. The paper by Verstraete (2018) - listed under Group III (beyond-inventory or methodological support) below-provides particular support for this group of authors by presenting a novel approach to regridding data. The paper by Oda et al. (2019) — listed under Group II (bottom-up/topdown accounting) below - makes use of the research reported by Bun et al. (2018).

The research of the authors in Group I is motivated by the desire to achieve a better territorial overview of where emissions appear locally. An increasing number of practical applications require knowledge of where emissions occur at increasingly smaller spatial scales.

However, high-resolution inventories come with much greater uncertainty. The reasons are discussed by Bun et al. (2018) and Hogue et al. (2017). There is uncertainty in the geolocation of emission sources/sinks; uncertainty resulting from the aggregation of statistical data; uncertainty underlying proxy and geolocational data; uncertainty regarding how proxy data, in the end, are represented; and last, but not least, uncertainty associated with the choice of emission factor.

The authors analyze the increase in uncertainty and develop methods to reduce it. The two approaches followed are (i) tracing emissions by source and estimating gridded totals (Bun et al. 2018; Charkovska et al. 2018, 2019; Danylo et al. 2019); and (ii) quantifying the uncertainty of gridded emissions as a function of grid size (Hogue et al. 2017).

The group of authors following the first approach (namely under [i]) does not start from a regular grid. Instead, emission (and removal) processes in all categories of human activity, as specified by the IPCC Guidelines for National Greenhouse Gas Inventories (IPCC 2006), are analyzed at the level of sources (and sinks). These are classified as point, line, or areal sources and according to intensity and physical size (with respect to the territory under investigation). The resulting geospatial database contains information about the administrative assignment of each emission source as a vector map object. 
The emissions from very diverse emission sources can be combined into a grid, allowing total emissions to be calculated, while the grid size can be chosen arbitrarily depending on analysis and visualization needs. This offers a unique opportunity to calculate total emissions for different levels of administrative unit (settlements, municipalities, districts, provinces) without loss of accuracy, as well as separately by emission category, greenhouse gas, type of fossil fuel, etc. The approach allows uncertainty to be considerably reduced for highresolution inventories. Starting by assembling statistical data from the lowest available administrative level (ideally municipality) limits disaggregation depth and errors. This is in contrast to handling uncertainty by way of disaggregation, which is applied, for example, in gridded approaches. Here, uncertainty is determined by disaggregation depth and increases with it, when going from large to smaller scales. Note that this important difference is preserved even if emissions estimated at, or aggregated to larger (e.g., national) scales closely agree.

Poland serves as a joint case study. Emissions from various sources are calculated both for grids and administrative units. The results demonstrate the considerable unevenness of spatial distributions of GHG emissions. Distributions and their uncertainty ranges are estimated by applying a Monte Carlo method. Bun et al. (2018) describe the approach in general; Charkovska et al. (2019) focus on emissions from industrial processes, Danylo et al. (2019) on emissions from the residential sector, and Charkovska et al. (2018) on emissions from agriculture.

The authors following the second approach (namely [ii]) look, in particular, at the uncertainty associated with the allocation of point sources. Any misallocation of these emission sources can have important consequences for high-resolution inventories, especially if their emission intensities are high. Such misallocations happen, for example, when databases are combined to merge information using logical rules. Hogue et al. (2017) analyze how the misallocation of point emission sources impacts inventory uncertainty as a function of grid size. The authors use population density as a proxy to distribute emissions spatially across grids that vary in size. They find that relative uncertainty (total uncertainty divided by total emissions) at grid-cell level decreases with increasingly coarser resolution. In most cases, relative uncertainty also decreases with increasing emissions from point sources. The authors' results indicate that good data of large point sources are particularly crucial for obtaining reliable, spatially explicit emission inventories.

\subsection{Group II: Bottom-up/top-down accounting (high resolution or gridded)}

The papers by Lesiv et al. (2018), Oda et al. (2019), and Zimnoch et al. (2018) explore the use of additional, ideally independent, observations as top-down constraints, such as atmospheric measurements and remote sensing, to identify and close potential inaccuracies in bottom-up inventories. Although, all three papers exercise bottom-up/top-down, high-resolution, or gridded, at subglobal scales, they show potential to expand to global scales - which is why, arguably, there are still some degrees of freedom in closing (verifying) bottom-up and topdown. These authors' research contributes substantially to key issue 1 (two-sided perspective) as well as to key issue 5 .

Lesiv et al. (2018) come up with a "verified" account of carbon in forest ecosystems over larger areas, in this case for Ukraine. The authors present a forest map for 2010 with a spatial resolution as high as $60 \mathrm{~m}$, which is needed to capture Ukraine's highly fragmented forest landscape. The forest map contains information about dominant tree species, total biomass, and net primary production (NPP). Together with forest inventory statistics and forest-related 
data collected by applying Geo-Wiki (https:/www.geo-wiki.org/) online, it allows Ukraine's net carbon flux for 2010 to be determined. To constrain that carbon flux, both a flux-based and a stock-based method are applied. The two methods indicate that Ukraine's forest serves as a net carbon sink in the range of $11.0 \pm 1.4$ (stock-based method) to $11.8 \pm 3.2 \mathrm{Tg} \mathrm{C} \mathrm{y}^{-1}$ (fluxbased method), or $11.4 \pm 1.7 \mathrm{Tg} \mathrm{C} \mathrm{y}^{-1}$ (equivalent to $131 \pm 20 \mathrm{~g} \mathrm{C} \mathrm{m}^{-2} \mathrm{y}^{-1}$ ) on average. This sink differs by bioclimatic zone, ranging from 55 in Polissya to $197 \mathrm{~g} \mathrm{C} \mathrm{m}^{-2} \mathrm{y}^{-1}$ in the Carpathians. Above and beyond determining uncertainty, an additional value of the authors' study is their demonstration of how to compensate for missing knowledge in the accounting of forest ecosystems by proceeding in a spatially explicit manner.

Oda et al. (2019) compare ODIAC (Open-source Data Inventory for Anthropogenic $\mathrm{CO}_{2}$ ) with GESAPU, a high-resolution, spatially explicit emission inventory-here, the one provided by Bun et al. (2018) for Poland. ODIAC is itself a global inventory with a spatial resolution of $1 \mathrm{~km} \times 1 \mathrm{~km}$, based on the disaggregation of the national annual fossil-fuel $\mathrm{CO}_{2}$ emission estimates provided by the Carbon Dioxide Information Analysis Center. To achieve that high spatial resolution, ODIAC uses point source information (source points' geographical location and $\mathrm{CO}_{2}$ emissions) and satellite nightlight (radiance) data. Because of its greater local "realism", GESAPU is used as a reference in this comparison. The difference between the two inventories is understood to serve as a proxy for errors and uncertainties associated with ODIAC. This difference is small for total emission estimates of countries $(2.2 \%)$, point sources $(0.1 \%)$, and non-point sources $(4.5 \%)$. However, it increases toward smaller spatial scales, indicating that disaggregation error and uncertainty increase. Oda et al. find a difference (relative at the pixel level) of typically about $30 \%$ for urban areas, up to $90-100 \%$ for urban-rural transition areas, and $10 \%$ for remote areas. The difference decreases with increasing spatial aggregation by approximately $70 \%$ for spatial scales, which are typical for global and regional transport models (50 km and greater). Based on their findings for Poland, the authors envisage using ODIAC globally to support monitoring verification and even at subnational levels - it is not unusual for countries to run emission inventories at the state or provincial levels while reporting only national emissions to the UNFCCC. However, as noted by the authors, such a request would need to accompany concerted global actions, ranging from the collection and reporting of data, through monitoring, to international governance.

Zimnoch et al. (2018) focus on top-down estimation of both $\mathrm{CO}_{2}$ and $\mathrm{CH}_{4}$ emissions from the urban area of Krakow, Poland. They present a set of methods based on atmospheric observations of $\mathrm{CO}_{2}$ and $\mathrm{CH}_{4}$ mixing ratios and their isotopic composition, the use of additional data relating to the atmospheric concentration of radon and mixing layer height, and atmospheric modeling, to identify and quantify urban emissions. These methods complement each other; they allow a determination not only of the contribution of different emission sources to the total atmospheric load but also of the fluxes of those gases. The methods provide an efficient way of quantifying the surface emissions of major GHGs from distributed sources and thus represent a complementary approach to accounting emission bottom-up. The authors' approach offers an alternative to validate the effectiveness of potential climate change mitigation strategies at scales of great interest to policy actors, demonstrated here for complex local urban-scale environments.

\subsection{Group III. Beyond-inventory or methodological support}

The papers by La Notte et al. (2018), Verstraete (2018), Gusti et al. (2018), Jarnicka and Żebrowski (2019), and Jonas and Żebrowski (2018) provide beyond-inventory or methodological support on aspects of uncertainty that have not been addressed to date, have been 
overlooked, or have emerged over time. The research described in these papers contributes equally to key issues 1 (one-sided perspective), 3, and 4.

La Notte et al. (2018) focus on combining, at the regional level, economic accounts with environmental data on atmospheric emissions (GHGs and air pollutants), with the atmospheric emissions combined into groups and expressed in terms of various potentials: their global warming potential (GWP), their potential acid equivalent (PAE), and their tropospheric ozone formation potential (TOPF). By considering uncertainty in an on-off mode (instantaneous learning), policy recommendations at regional and provincial levels can be made.

Verstraete (2018) proposes a new method that does not lead to an increase in uncertainty during the process of overlaying data sets mapped to different grids. This so-called regridding process is an important preprocessing tool in handling spatially resolved datasets, offering considerable potential, particularly for authors compiling high-resolution spatial inventories (cf. Group I). Such researchers frequently face the problem of having to rely on data represented using different grids (e.g., proxy data). These can differ in terms of cell size, or they can be displaced latitudinally and/or longitudinally or even be rotated relative to each other; and grids can also be irregular. The approach offered by Verstraete can be used for remapping, for example, a grid onto administrative borders (or vice versa). Fuzzy rule-based methods are elaborated and tested for regridding using additionally available knowledge in order to obtain better results, particularly during spatial disaggregation processes.

Gusti et al. (2018) address the uncertainty underlying marginal abatement cost curves (MACCs) derived for the LULUCF sector, by studying their sensitivity to uncertainty in the price of agricultural land and forestry commodities and to uncertainty in the quality of governance - stability, effectiveness, and assertiveness (simplified and summarized by means of a so-called "corruption" coefficient) — in the regions studied, ranging from individual countries to the global level. The uncertainty in MACCs, if not considered, may crucially influence the trade of emission permits or undermine decisions based on MACCs to mitigate GHG emissions. The authors' results indicate that MACCs are especially more sensitive to the quality of governance than to the price of agricultural land. MACCs appear more robust for high $\mathrm{CO}_{2}$ prices, while they are more sensitive to the variation in these parameters for low $\mathrm{CO}_{2}$ prices. The authors conclude that considering the quality of governance is key if medium-term mitigation policies, usually designed for low $\mathrm{CO}_{2}$ prices, are developed.

Jarnicka and Żebrowski (2019) quantify (historical or diagnostic) learning - defined as the decrease in uncertainty (inaccuracy and imprecision) in the estimates of GHG emissions with the focus here on country $\mathrm{CO}_{2}$ emission totals (excluding LULUCF emissions) - reported in national inventory reports. The authors demonstrate how knowledge of a change in uncertainty can be gained from analyzing annually revised emission estimates in retrospect. In cases of pronounced learning, the reduction in uncertainty can be well described by the coefficient in an exponential model. The authors' results allow the conclusion to be drawn that continuous efforts (i.e., time series of 20 years and longer of continuously revised emissions) are necessary to determine a half-time of this reduction (of the order of 5 years for the EU15) that is sufficiently robust. Their approach goes beyond that favored by the IPCC of estimating and monitoring uncertainty to help prioritize efforts to improve the accuracy of inventories and guide decisions on methodological choice (IPCC 2000: Chapter 6). Understanding what it takes to decrease uncertainty over time is crucial, on the one hand for evaluating the quality of compliance under which countries meet their emission reduction targets and, on the other, for setting future emission reduction targets more skillfully, that is, from an emission changeversus-uncertainty perspective rather than from an emissions change-only perspective. 
Jonas and Żebrowski (2018) focus on the memory and persistence of forced, causally linked systems - such as population, GHG emissions, atmospheric concentrations, and average surface air temperature - with GHG emissions into the atmosphere serving as their case in point (although the authors still prefer working with synthetic data). Memory allows reference to be made to how strongly the past can influence the "near-term future" of the system, its socalled explainable outreach. In contrast to Jarnicka and Żebrowski, the mode of learning underlying these authors' approach to analyzing data in retrospect could be termed "diagnostic learning under controlled prognostic conditions". (Data are subdivided for testing and learning.) In light of the continued increase in emissions globally vis-à-vis, the reductions urgently needed until 2050 and beyond, the authors conjecture that, being ignorant of memory and persistence, the "inertia" with which global GHG emissions will continue on their increasing path beyond today is underestimated and thus, that the amount of reduction achievable in the future is overestimated. This inertia is initially caused by human behavior (leading to emissions of GHGs into the atmosphere), but it will become increasingly "geophysical" the more Earth processes (e.g., the manifestation of emissions as concentrations of GHGs in the atmosphere) are disturbed. The authors anticipate that persistence is a powerful system characteristic, by which they mean that the system's explainable outreach appears determinable even under incomplete knowledge of memory and imperfect understanding of how the system is forced.

\section{Summary}

The approaches to addressing uncertainty discussed in this special issue represent an attempt to improve national inventories, not only for their own sake but also from a wider, system analytical perspective that seeks to strengthen their usefulness under a compliance and/or global monitoring and reporting framework. They thus reflect the challenges and benefits of including inventory uncertainty in policy analysis, and where advances are being made. The issues raised by the authors and featured in their papers, and the role played by uncertainty analysis in many of their arguments, highlight the importance of such efforts. While the IPCC clearly stresses the value of conducting uncertainty analyses and offers guidance on executing them, the arguments made here in favor of studying uncertainty go well beyond any suggestions made by the IPCC to date. The rationale recalled in Box 2 for improving and conducting uncertainty analyses is considered to still hold true: uncertainty analysis is needed for developing clear understanding and informed policy. Uncertainty matters and it is key to many issues related to inventorying and reducing emissions. Dealing proactively with uncertainty allows useful knowledge to be generated that the international community should have to hand while strengthening the 2015 Paris Agreement, the successor agreement to the Kyoto Protocol.

From a systemic perspective, we refer to six important insights/issues to reflect our status quo understanding of uncertainty research - (1) verification; (2) avoidance of systemic surprises; (3) uncertainty informing policy; (4) minimizing the impact of uncertainty; (5) full GHG accounting; and (6) compliance versus reporting. All papers in this special issue confirm or advance key insights 1, 3, 4, and 5. None of the papers addresses key insights 2 or 6-which does not come as a surprise. Anticipating systemic surprises is not easy. The same holds for tailoring international agreements cleverly (e.g., by way of bifurcation), which requires an advanced understanding of the entire GHG emission system. We expect that key insights 2 and 6 will receive greater attention in the near future. 
Nonetheless, the authors demonstrate that great strides can be made in improving national inventories of GHG emissions by considering uncertainty in the emissions. For instance, biases can be reduced resulting in more accurate emission estimates; uncertainty can be decreased, or kept small, while emission sources (and sinks) are spatially disaggregated; and emission reduction policies can be made more robust by understanding the sources of uncertainty.

Yet, as the reader will understand when going through the various papers in this special issue, many important questions remain. Questions of a scientific nature may prevail at first glance. However, all authors are guided by a principal question: "verifying what GHGs at what spatio-temporal scale will support conducive legislation at local and national scales while ensuring effective governance at the global scale?"

We hope that a wide community will enjoy reading the papers in this special issue.

Acknowledgments Open access funding provided by International Institute for Applied Systems Analysis (IIASA). The authors would like to thank Zbigniew Nahorski, Joanna Horabik, Jolanta Jarnicka, and Weronika Radziszewska from the Systems Research Institute of the Polish Academy of Sciences for organizing the 4th International Workshop on Uncertainty in Atmospheric Emissions; and Kathryn Platzer and Ansa Heyl associated with IIASA's External Relations, Communications, and Library Department for their support and editorial work in publishing this special issue.

Open Access This article is distributed under the terms of the Creative Commons Attribution 4.0 International License (http://creativecommons.org/licenses/by/4.0/), which permits unrestricted use, distribution, and reproduction in any medium, provided you give appropriate credit to the original author(s) and the source, provide a link to the Creative Commons license, and indicate if changes were made.

\section{References}

Bun R, Nahorski Z, Horabik-Pyzel J, Danylo O, See L, Charkovska N, Topylko P, Halushchak M, Lesiv M, Valakh M, Kinakh V (2018) Development of a high-resolution spatial inventory of greenhouse gas emissions for Poland from stationary and mobile sources. Mitig Adapt Strateg Glob Chang https://link.springer. com/article/10.1007\%2Fs11027-018-9791-2. https://doi.org/10.1007/s11027-018-9791-2. Accessed 23 Feb 2018

Charkovska N, Horabik-Pyzel J, Bun R, Danylo O, Nahorski Z, Jonas M, Xiangyang X (2018) High resolution spatial distribution and associated uncertainties of greenhouse gas emissions from the agricultural sector. Mitig Adapt Strateg Glob Chang https://link.springer.com/article/10.1007\%2Fs11027-017-9779-3. https://doi.org/10.1007/s11027-017-9779-3. Accessed 11 Jan 2018

Charkovska N, Halushchak M, Bun R, Nahorski OT, Jonas M, Topylko P (2019) A high-definition spatially explicit modelling approach for national greenhouse gas emissions from industrial processes: reducing the errors and uncertainties in global emission modelling. Mitig Adapt Strateg Glob Change https://link.springer. com/article/10.1007\%2Fs11027-018-9836-6. https://doi.org/10.1007/s11027-018-9836-6. Accessed 10 Jan 2019

Danylo O, Bun R, See L, Charkovska N (2019) High resolution spatial distribution of greenhouse gas emissions in the residential sector. Mitig Adapt Strateg Glob Chang https://link.springer.com/article/10.1007 \%2Fs11027-019-9846-z. https://doi.org/10.1007/s11027-019-9846-Z. Accessed 25 March 2019

Gusti M, Forsell N, Havlik P, Khabarov N, Kraxner F, Obersteiner M (2018) The sensitivity of the costs of reducing emissions from deforestation and degradation (REDD) to future socioeconomic drivers and its implications for mitigation policy design. Mitig Adapt Strateg Glob Chang https://link.springer. com/article/10.1007\%2Fs11027-018-9817-9. https://doi.org/10.1007/s11027-018-9817-9. Accessed 08 June 2018

Hogue S, Roten D, Marland E, Marland G, Boden T (2018) Gridded estimates of $\mathrm{CO}_{2}$ emissions: uncertainty as a function of grid size. Mitig Adapt Strateg Glob Chang https://ink.springer.com/article/10.1007\%2Fs11027017-9770-z. https://doi.org/10.1007/s11027-017-9770-z. Accessed 08 Dec 2017 
IPCC (2000) Good practice guidance and uncertainty management in national greenhouse gas inventories. Institute for Global Environmental Strategies (IGES), Hayama. https://www.ipcc-ggip.iges.or. jp/public/gp/english/

IPCC (2006) 2006 IPCC Guidelines for national greenhouse gas inventories. Institute for Global Environmental Strategies, Hayama. https://www.ipcc-nggip.iges.or.jp/public/2006gl/

Jarnicka J, Żebrowski P (2019) Learning in greenhouse gas emission inventories in terms of uncertainty improvement over time. Mitig Adapt Strateg Glob Chang

Jonas M, Żebrowski P (2018) The crux with reducing emissions in the long-term: the underestimated "now" versus the overestimated "then". Mitig Adapt Strateg Glob Chang https://ink.springer.com/article/10.1007 \%2Fs11027-018-9825-9. https://doi.org/10.1007/s11027-018-9825-9. Accessed 21 Sept 2018

La Notte A, Tonin S, Nocera S (2018) A screening procedure to measure the effect of uncertainty in air emission estimates. Mitig Adapt Strateg Glob Chang https:/link.springer.com/article/10.1007\%2Fs11027-018-97988. https://doi.org/10.1007/s11027-018-9798-8. Accessed 22 March 2018

Lesiv M, Shvidenko A, Schepaschenko D, See L, Fritz S (2018) A spatial assessment of the forest carbon budget for Ukraine. Mitig Adapt Strateg Glob Chang https://link.springer.com/article/10.1007\%2Fs11027-0189795-y. https://doi.org/10.1007/s11027-018-9795-y. Accessed 16 March 2018

NRC (2010) Verifying greenhouse gas emissions: methods to support international climate agreements. National Research Council of the National Academies, Washington DC https://www.nap.edu/catalog/12883 /verifying-greenhouse-gas-emissions-methods-to-support-international-climate-agreements

Oda T, Bun R, Kinakh V, Topylko P, Halushchak M, Marland G, Lauvaux T, Jonas M, Maksyutov S, Nahorski Z, Lesiv M, Danylo O, Horabik-Pyzel J (2019) Errors and uncertainties in a gridded carbon dioxide emissions inventory. Mitig Adapt Strateg Glob Chang

Ometto JP, Bun R, Jonas M, Nahorski Z (eds) (2015) Greenhouse gas inventories: expanding our uncertainty perspective. Springer, Dordrecht

Tollefson J (2018a) Can the world kick its fossil-fuel addiction fast enough? Nature 556:422-425 https://www. nature.com/articles/d41586-018-04931-6

Tollefson J (2018b) Nations must triple efforts to curb greenhouse-gas emissions. Nature News https://www. nature.com/articles/d41586-018-07566-9. https://doi.org/10.1038/d41586-018-07566-9

UNFCCC (2018) Understanding the UN climate change regime. UNFCCC Secretariat, Bonn https://unfecc. int/resource/bigpicture/\#content-understanding-the-un-climate-change-regime

Verstraete J (2018) Solving the general map overlay problem using a fuzzy inference system designed for spatial disaggregation. Mitig Adapt Strateg Glob Chang https://link.springer.com/article/10.1007\%2Fs11027-0189823-y. https://doi.org/10.1007/s11027-018-9823-y. Accessed 10 July 2018

Weiss RF, Prinn RG (2011) Quantifying greenhouse-gas emissions from atmospheric measurements: a critical reality check for climate legislation. Phil Trans R Soc A 369:1925-1942 https://royalsocietypublishing. org/doi/full/10.1098/rsta.2011.0006

White T, Jonas M, Nahorski Z, Nilsson S (eds) (2011) Greenhouse gas inventories: dealing with uncertainty. Springer, Dordrecht

Zimnoch M, Necki J, Chmura L, Jasek A, Jelen D, Galkowski M, Kuc M, Gorczyca Z, Bartyzel J, Rozanski K (2018) Quantification of carbon dioxide and methane emissions in urban areas: source apportionment based on atmospheric observations. Mitig Adapt Strateg Glob Chang https://link.springer.com/article/10.1007 \%2Fs11027-018-9821-0. https://doi.org/10.1007/s11027-018-9821-0. Accessed 12 June 2018

Publisher's note Springer Nature remains neutral with regard to jurisdictional claims in published maps and institutional affiliations. 


\section{Affiliations}

\section{Matthias Jonas ${ }^{1} \cdot$ Rostyslav Bun ${ }^{2,3} \cdot$ Zbigniew Nahorski $^{4} \cdot$ Gregg Marland $^{5}$. Mykola Gusti $^{1,2} \cdot$ Olha Danylo $^{1}$}

$\triangle$ Matthias Jonas

jonas@iiasa.ac.at

Rostyslav Bun

Rostyslav.A.Bun@lpnu.ua

Zbigniew Nahorski

Zbigniew.Nahorski@ibspan.waw.pl

Gregg Marland

marlandg@appstate.edu

Mykola Gusti

gusti@iiasa.ac.at

Olha Danylo

danylo@iiasa.ac.at

International Institute for Applied Systems Analysis, Laxenburg, Austria

2 Lviv Polytechnic National University, Lviv, Ukraine

3 WSB University, Dąbrowa Górnicza, Poland

4 Systems Research Institute, Polish Academy of Sciences, Warsaw, Poland

5 Appalachian State University, Boone, NC, USA 